\title{
Lire ou ne pas (pouvoir) lire. Marque satanique, appareil judiciaire et ambiguités herméneutiques chez Kafka
}

Lesen oder nicht lesen (können). Satanisches Körpermal, Justizwesen und hermeneutische Ambiguitäten bei Kafka.

To read or not (be able) to read. Satanic mark, judicial apparatus and hermeneutical ambiguities in Kafka's work

\section{Anne Isabelle François}

\section{OpenEdition}

\section{Journals}

Édition électronique

URL : https://journals.openedition.org/ceg/11107

DOI : $10.4000 /$ ceg. 11107

ISSN : 2605-8359

Éditeur

Presses Universitaires de Provence

Édition imprimée

Date de publication : 1 octobre 2012

Pagination : 441-453

ISBN : 0751-4239

ISSN : 0751-4239

Référence électronique

Anne Isabelle François, « Lire ou ne pas (pouvoir) lire. Marque satanique, appareil judiciaire et ambiguïtés herméneutiques chez Kafka », Cahiers d'Études Germaniques [En ligne], 63 | 2012, mis en ligne le 07 septembre 2020, consulté le 04 juin 2021. URL : http://journals.openedition.org/ceg/11107 ; DOI : https://doi.org/10.4000/ceg.11107 


\title{
Lire ou ne pas (pouvoir) lire Marque satanique, appareil judiciaire et ambiguïtés herméneutiques chez Kafka
}

\author{
Anne Isabelle FRANÇOIS \\ Université Sorbonne Nouvelle-Paris 3
}

Eine Spur ist die Erscheinung einer Nähe,

so fern sie sein mag.

Walter Benjamin

Alle diese Gleichnisse wollen eigentlich nur sagen, dass das Unfassbare unfassbar ist,

und das haben wir gewusst.

Franz Kafka

I. C'est une banalité de dire que l'île de la Colonie pénitentiaire est proprement infernale : chaudron tropical brûlé par un soleil impitoyable ${ }^{1}$, gouverné par une secte avec ses propres textes sacrés et un démiurge dont on prophétise le retour après lui avoir refusé une sépulture dans le cimetière ${ }^{2}, \mathrm{y}$

1 L'ensemble se passe dans un vallon désolé, abrupt et sablonneux, cerné de pentes dénudées. Le texte souligne explicitement le feu du ciel, dont souffre au moins "le chercheur en voyages d'études" (Forschungsreisender): "die Sonne verfing sich allzustark in dem schattenlosen Tal", "die Augen schmerzten ihn von dem mit Sonnenlicht überschütteten Himmel". L'officier à l'inverse, pourtant solidement sanglé dans son sévère uniforme, ne semble guère importuné par cette fournaise - ce qui pointe vers sa "nature" indigène sinon diabolique. Il n'est pas jusqu'à la machine qui étincelle de manière inquiétante, comme les "quatre montants de cuivre jaune qui, au soleil, lançaient presque des rayons" ("vier Messingstangen [...], die in der Sonne fast Strahlen warfen"). Franz KAFKA, In der Strafkolonie, in Franz KAFKA, Erzählungen, éd. M. Müller, Stuttgart, Reclam, 1995, p. 133 , 156, 135 ; Franz KAFKA, Dans la colonie pénitentiaire, trad. B. Lortholary, Paris, GF, 1991, p. 90. Toutes nos citations renvoient à ces éditions.

2 L'ancien commandant, génie tout-puissant à qui l'on doit l'invention de la machine et de toute l'organisation de la colonie, tout à la fois "soldat, juge, technicien, chimiste, dessinateur" (p. 91 ; "Hat er denn alles in sich vereinigt ? War er Soldat, Richter, Konstrukteur, Chemiker, 
trône une machine judiciaire de torture, "un appareil singulier"3 d'une inénarrable cruauté. C'est pourtant un autre aspect qui va être au centre de cette réflexion, plus subtil, mais non moins prégnant et essentiel à la célèbre nouvelle (In der Strafkolonie, 1919), qui permet d'y voir le point d'aboutissement d'un vaste héritage doctrinal et spéculatif autant qu'imaginaire. Kafka pousse à son terme, à son ultime limite, mais en le brouillant et en le faisant dérailler à l'instar de son "appareil singulier", l'ancienne croyance en l'existence d'une marque satanique. Signe de reconnaissance et trace distinctive, dévoilant l'identité de l'homme, cette marque constituerait la signature pour ainsi dire originelle du diable, au sens propre sa "griffe" . Kafka la réinvestit ici dans une optique toute moderne, selon le principe que tout indice et signe est tributaire de codes culturels, eux aussi tributaires de l'époque, qui fixent dans l'imaginaire les motifs et images dans un rôle bien déterminé.

C'est ce processus, à la fois de naturalisation et de sécularisation, que nous nous proposons d'étudier, plus précisément l'hypothèse d'un processus de laïcisation contradictoire qui revient à se réapproprier tout en le brouillant le topos de la marque diabolique. La parabole de Kafka opère et exprime ce tournant sémiotique qui installe l'idée d'une grammaire des signes sataniques, entendant constituer un corps éminemment marqué, miroir de l'âme, tout en se heurtant à l'aporie d'un objet toujours irrémédiablement opaque. Ce processus a par ailleurs expressément à voir avec le judiciaire d'une part c'est-à-dire avec la question du pouvoir, des rapports de force, au sens proprement d'emprise -, avec l'écriture de l'autre, à travers le fantasme de la signature corporelle, d'une peau comme texte rendant visible / lisible le caché, comme surface d'inscription et d'affleurement où lire et interpréter les signes. Le fantasme est donc à cet égard indissociable du discours scientifique - anthropométrie, physiognomonie - et de la machine de l'identification judiciaire, la peau fonctionnant comme le miroir magique des contes : lieu où s'inscrivent et s'extériorisent les traces de l'abomination, de la dépravation, de la faute, elle révèle l'âme.

Ce qui est ici en jeu est en somme une dialectique compliquée de la surface et de la profondeur, de la transparence et de l'opacité, de la distance et de la proximité. Entrer en contact avec Satan, ce serait être marqué dans sa chair, le diable signant ceux qui se donnent à lui d'un stigmate explicite, le punctum ou stigma diabolicum ${ }^{5}$. Mais les textes anciens rappellent également

Zeichner ?", p. 136), est l'auteur de précieux dessins à l'écriture compliquée, chargée d'enjolivures et accessible qu'aux seuls initiés, que ne peuvent manipuler, qu'avec dévotion à l'instar d'un texte sacré, que les plus fidèles parmi ses disciples (Anhänger).

3 P. 85 ; "ein eigentümlicher Apparat" (p. 131).

4 Selon la formule de Gwenhaël PONNAU, "La griffe et les oripeaux du diable (fantastique et démonomanie)", in Les Arts du Diable. Revue des Sciences Humaines, avril-juin 1994, $\mathrm{n}^{\circ} 234$, p. $85-96$.

5 La marque, témoignage visible de l'alliance, directement imprimée par le diable au moyen d'un instrument ou, plus souvent, avec l'une de ses griffes, est, dans l'imaginaire démonologique, l'équivalent d'une signature par laquelle Satan s'assure la fidélité et 
de manière insistante la difficulté à trouver cette trace ; on se heurte ainsi à l'absence perpétuelle d'une "empreinte" insensible, inassignable souvent et sans cesse vagabonde, Satan donnant la plupart du temps des marques "à cachette" (dissimulées). D'où le paradoxe d'une marque sur le corps à la fois indubitable et cachée, d'une peau qui donne à voir tout en masquant, où il s'agit de compenser la dénudation radicale par l'exhibition d'un scriptible qui soit aussi lisible. C'est la diffraction de ce discours du fantasme dans l'imaginaire et en particulier le texte littéraire, bien après le discrédit de la chasse aux sorcières, que nous allons examiner. La littérature s'empare du paradigme du corps comme texte ambigu, d'un corps marqué sur lequel on pourrait lire à livre ouvert, d'un corps qui montre l'imprégnation "diabolique" à l'aide de signes et de traces ostensibles mais qui demandent pourtant à être révélés - sans peut-être que cela soit possible. La nouvelle de Kafka exprime ainsi de manière exemplaire le paradoxe, réinvestissant, peu ou prou, l'antique science démonologique et physiognomonique, en la sécularisant, retravaillant dans une constellation inédite, et atroce, la dialectique de la profondeur et de la surface, transformant la texture démoniaque en véritable piège interprétatif.

II. Tout le monde se livre à une entreprise de déchiffrement dans la nouvelle de Kafka, s'efforçant de lire des signes qui paraissent tout sauf indiscutables. Le condamné ne cesse ainsi d'essayer de comprendre la fonction de la machine et ce que s'en disent l'officier et le voyageur, mais sans y parvenir, "puisque l'explication lui manquait"'. L'officier cherche, dit explicitement le texte, à "lire", sans doute en vain, sur le visage de son interlocuteur, ce chercheur dont on nous précise qu'il ne voyage "que dans l'intention de voir" ${ }^{8}$. Le déchiffrement même de l'inscription sur la tombe de l'ancien commandant ne va pas sans difficultés, non seulement parce que cette tombe elle-même est "dissimulée" sous les tables de thé, mais aussi parce que l'inscription elle-même y est gravée "en très petits caractères" et que le voyageur doit s'agenouiller pour la lire? . C'est enfin l'écriture de l'ancien commandant qui s'avère la plus difficile d'accès, ces "lignes

l'obéissance de ceux qui, comme des infâmes ou des esclaves marqués au fer par leur maître, sont définitivement et radicalement distingués de la communauté chrétienne : exclus et enfermés à la fois.

6 P. 97 : "[...] wie es ihm aber, da ihm die Erklärung fehlte, nicht gelingen wollte [...]" (p. 140). Cf. p. 143 : "Auch der Verurteilte sah ihr zu, aber ohne Verständnis" (p. 143). "Voir" ne suffit donc pas pour "comprendre" (begreifen).

7 P. 101 : "Der Offizier sah ununterbrochen den Reisenden von der Seite an, als suche er von seinem Gesicht den Eindruck abzulesen, den die Exekution, die er ihm wenigstens oberflächlich erklärt hatte, auf ihn mache" (p. 143 ; je souligne).

8 P. 102-103: "denn er reise nur mit der Absicht zu sehen" (p. 144).

9 P. 126 ; "Es war ein einfacher Stein, niedrig genug, um unter einem Tisch verborgen werden zu können. Er trug eine Aufschrift mit sehr kleinen Buchstaben, der Reisende musste, um sie zu lesen, niederknien" (p. 162 ; je souligne). 
labyrinthiques qui s'entrecroisaient en tous sens et couvraient le papier de manière si dense qu'on avait peine à discerner des blancs entre elles"10.

Tous les personnages en somme mettent en œuvre le "paradigme indiciaire" ${ }^{11}$ où, à partir d'indices minimaux, ils entendent avoir accès à la vérité par l'interprétation. En l'occurrence, cette herméneutique passe par le repérage d'indices minimaux, de traces infimes - et c'est aussi dans ce contexte qu'entre le phénomène d'étiquetage et de marquage entrepris par la machine. Plus précisément son objet, comme dans le paradigme indiciaire, est de rendre visible ce qui est invisible, c'est-à-dire d'extérioriser et d'expliciter ce qui est intérieur : la faute du condamné, qui lui est gravée sur le corps. Il s'agit même d'annuler quasiment la différence entre surface et profondeur, cette dialectique étant travaillée tout au long de la nouvelle : la sentence du condamné, indubitable puisque "la culpabilité ne fait jamais de doute"12, affleure à la surface, est inscrite sur sa peau dénudée. Mais cette inscription se fait couche après couche, de manière à également l'enfoncer dans ce corps, à l'y enfouir de nouveau, comme l'expose l'officier, jusqu'à la fin de la procédure : "Justice est faite, alors, et le soldat et moi nous l'enfouissons"13.

L'inscription sur le corps du condamné de sa sentence, ou plutôt de la règle qu'il a enfreinte, est donc bien le signe tangible d'une faute, d'une culpabilité ; la peau fonctionne comme texte et comme preuve. C'est aussi pourquoi l'officier tente de "lire" sur le visage du voyageur, cette "fenêtre" de l'âme. Kafka réinvestit ainsi ici le fantasme traditionnel d'un corps "expressif", d'une peau-texte qui renvoie autant à celui qui l'a marquée qu'à son porteur, selon le principe du miroir - qu'il s'agisse de signes "de naissance" ou de signes "acquis", comme les cicatrices et autres tatouages ${ }^{14}$.

10 P. 98 ; "er sah nur labyrinthartige, einander vielfach kreuzende Linien, die so dicht das Papier bedeckten, dass man nur mit Mühe die weißen Zwischenräume erkannte" (p. 141).

11 Cf. Carlo Ginzburg, "Traces. Racines d'un paradigme indiciaire" [1979], trad. M. Aymard, in Carlo GinzBurg, Mythes, emblèmes, traces. Morphologie et histoire, nouvelle éd. augmentée, Lagrasse, Verdier poche, 2010, p. 218-294. Sur ce point, voir aussi Jacques RANCIÈRE, "De la vérité des récits au partage des âmes", Critique n 769-770 ("Sur les traces de Carlo Ginzburg"), éd. Patrizia Lombardo et Martin Rueff, Paris, Minuit, juin-juillet 2011, p. 474-484.

12 P. 93 ; "Die Schuld ist immer zweifellos" (p. 137).

13 P. $100 ;$; "...] und zu neuer Vertiefung der Schrift vorbereitet. [...] So schreibt sie immer tiefer die zwölf Stunden lang. [...] Dann ist das Gericht zu Ende, und wir, ich und der Soldat, scharren ihn ein" (p. 142-143; je souligne). On retrouve la tension dans la tentative interprétative de l'officier pour lire sur le visage du voyageur sa réaction : il s'agit d'y avoir accès la profondeur (ses pensées), alors que son explication n'a pu être que superficielle (oberflächlich, cf. citation de la note 7).

14 Cf. Jane CAPlan (éd.), Written on the Body: The Tattoo in European and American History, Londres, Reaktion Books, 2000. Erich Auerbach n'ouvre-t-il pas de même sa magistrale étude sur l'épisode d'Ulysse et de la nourrice où c'est précisément la cicatrice qui tient lieu de signe de reconnaissance (Erich AuERBACH, Mimesis. Dargestellte Wirklichkeit in der europäischen Literatur, $2^{\mathrm{e}}$ éd., Berne, Francke, 1959, p. 7 sq.) ? Sur la présence du thème dans la littérature moderne de langue allemande : Gerard BARTL, Spuren und Narben. Die Fleischwerdung der Literatur im Zwanzigsten Jahrhundert, Würzburg, Königshausen \& Neumann, 2002 (sur Kafka : p. 191-274). 
Cet invariant de la culture occidentale repose sur l'idée que les traits extérieurs manifestent les traits intérieurs, en particulier les vices diaboliques. Sur la scène élisabéthaine, c'est ainsi à l'aide des furoncles et taches de naissance que le public identifie aisément la véritable identité d'un spectre comme dans le Doctor Faustus (1592) de Christopher Marlowe ${ }^{15}$.

Or cet invariant a connu la même mutation que la représentation et l'image même du diable qui, comme le note par exemple Daniel Arasse dans sa belle étude, a, sous l'influence de l'humanisme, été à la fois humanisé, intériorisé et sécularisé ${ }^{16}$. Le principe d'un visage et d'un corps sur lequel lire la réalité de l'âme, et plus précisément le sceau du vice, les stigmates de la dépravation, le signe du péché, était trop puissant et riche pour que l'imaginaire occidental s'en défasse ou l'abandonne ; on assiste donc à un processus de "naturalisation" à partir de la Renaissance au moins. Réinvestissant notamment l'idée d'un lien naturel entre le mal et la laideur, identification que Platon a reprise au niveau réflexif ${ }^{17}$, réinterprétant le "signe de Caïn" de la Genèse (4.15), réactivant l'antique science physiognomonique, se laïcise la conviction d'une signature matérielle sur la peau du magicien et de la sorcière.

Ainsi un glissement rapide s'opère du physique au moral : la marque visible sur la peau devient le signe de l'imperfection; elle est interprétée comme une tache, une souillure, le signe du péché et du mal, le révélateur de l'élection et emprise sataniques. C'est bien cette symbolique qui est à l'œuvre dans la nouvelle de Kafka - bien que compliquée par le déraillement de l'appareillage judiciaire sur lequel nous aurons à revenir - tout comme dans nombre de fictions contemporaines. Ainsi le portrait de Dorian Gray, dans le roman éponyme de Wilde (1890), change, on le sait, avec le temps, portant de façon visible les signes des dépravations et du vieillissement du héros : les traits mouvants du tableau peint révèlent la dégradation véritable de sa vie, le péché s'inscrivant sur le visage. Les marques sur le tableau, qui s'opposent à la figure "réelle" immaculée du personnage, sont bien les signes tangibles de Satan, les "signes du mal", une "tache" dit le texte ${ }^{18}$. Gray est à cet égard l'équivalent de la face d'Edward Hyde dans la nouvelle de Stevenson (1886), sur laquelle le notaire identifie explicitement la "griffe" diabolique : "si jamais j'ai vu la signature de Satan (Satan's signature) sur un visage, c'est sur celui de votre nouvel ami !",

15 Sur ce point : Stephen Greenblatt, Hamlet in Purgatory, Princeton (New Jersey), Princeton UP, 2001, p. 156.

16 Cf. Daniel ARASSE, Le portrait du Diable, [1989], Paris, Arkhê, 2009.

17 Il écrit ainsi dans les Lois : "Nécessairement, la vertu est belle et le vice laid" (900c). Voir aussi Gilbert LASCAULT, Le Monstre dans l'art occidental. Un problème esthétique, Paris, Klincksieck, 1973, p. 194-305.

18 Oscar Wilde, Le Portrait de Dorian Gray, trad. J. Gattégno, Paris, Gallimard, "Folio classique", 1992, p. 183 : "À chaque péché commis, une tache (a stain) viendrait souiller et ruiner sa beauté".

19 Robert Louis STEVenson, The Strange Case of Dr Jekyll and Mr Hyde / L'étrange cas du Dr Jekyll et M. Hyde, trad. C. Ballarin, Paris, Gallimard, "Folio bilingue", 1992, p. 60. 
Faces marquées sur lesquelles on peut lire à livre ouvert la biographie des êtres et de leurs abominations, le portrait de Dorian Gray et Mr. Hyde incarnent bien le principe du visage comme miroir de l'âme ${ }^{20}$. Citons enfin un dernier exemple symptomatique, en raison de sa proximité avec la parabole de Kafka : La tache de naissance de Nathaniel Hawthorne (The Birthmark, 1843). La jeune épouse du scientifique Aylmer, Georgiana, y est affligée, au centre de sa joue gauche, d'une marque de naissance qui ressemble à une petite main. Cette marque "de sang" sur sa peau immaculée, "tache écarlate sur la neige ${ }^{" 21}$, finit par obséder Aylmer, devenant à ses yeux le signe même de son imperfection: "le symbole de la vulnérabilité de sa femme face au péché, au chagrin, au vieillissement, et à la mort [...] le symbole de l'imperfection"22. Il entreprend donc de trouver la formule chimique qui lui permettra de restaurer une apparence parfaite à son épouse - quel qu'en soit le risque. Or le point intéressant est que, comme chez Kafka, l'ensemble de la nouvelle tourne autour de la dialectique profondeur/surface et que leurs statuts respectifs sont dorénavant devenus indiscernables. Tout comme l'aiguille de "l'appareil singulier" dans la colonie pénitentiaire entre de plus en plus profondément dans la peau du condamné, Aylmer se doit de trouver une potion qui lui permette d'éliminer la tache odieuse sur la joue de sa femme non seulement en surface, mais bien en profondeur - la tuant au moment où il $\mathrm{y}$ parvient $\mathrm{t}^{23}$.

III. Le principe de la marque satanique que réinvestit ainsi, laïcisé, la littérature moderne, a une origine clairement théologique, plus précisément démonologique. Il prend naissance à la Renaissance, lorsque s'impose la théorie d'une contre-société satanique en train de définitivement conquérir le monde et de renverser l'ordre chrétien, donnant lieu à une immense "explosion discursive" ${ }^{24}$ et produisant un savoir cumulatif presque sans

20 Conan Doyle fut d'ailleurs tellement impressionné par Dorian Gray et le thème du visage détruit, autre manière de faire surgir dans le réel la crainte de "perdre la face", qu'il le reprit dans une de ses nouvelles, "Le Client célèbre" (The Adventure of the Illustrious Client, 1924). On pensera également à la fin des Liaisons dangereuses (1782) où la Marquise de Merteuil perd elle aussi "la face" ; défigurée par la petite vérole, son visage correspond enfin à son âme.

21 Nathaniel Hawthorne, La marque de naissance, in Contes et récits, trad. M. Zagha, Arles, Actes Sud, Babel, 1996, p. 245 ; The Birthmark, in Nathaniel Hawthorne's Tales, éd. J. McIntosh, New York, Norton, 1987, p. 119 : "a crimson stain upon the snow". Hawthorne varie ici clairement le motif féerique de Blanche-Neige, cette jeune fille à la peau blanche comme la neige et aux lèvres et joues rouges comme le sang.

22 Ibid., p. 246 ; "the fatal flaw of humanity [...] the symbol of his wife's liability to sin, sorrow, decay, and death [...] the symbol of imperfection" (p. 120).

23 Lorsque Georgiana entre pour la première fois dans son laboratoire et y aperçoit une fiole, son mari lui répond : “ce produit n'agit qu'en surface. Ton cas requiert un remède plus pénétrant" (p. 256); "this is merely superficial. Your case demands a remedy that shall go deeper" (p. 125 ; je souligne).

24 Michel Foucault emploie l'expression dans son Histoire de la sexualité (Michel FoucAult, La volonté de savoir, Paris, Gallimard, "tel", 1978, p. 25-67) pour étudier, à l'encontre de 1" "hypothèse répressive", la prolifération des discours que, depuis trois siècles, le 
commune mesure. L'élaboration de la notion de marque sorcellaire est la réactivation, aux $\mathrm{XVI}^{\mathrm{e}}$ et $\mathrm{XVII}^{\mathrm{e}}$ siècles, de l'antique science physiognomonique, application au corps de la théorie des signatures : tout individu est censé porter, sous la forme de traits ou marques visibles, la trace de ses principales caractéristiques psycho-physiologiques ; son "tempérament", les affinités, sympathies et analogies qu'il détermine entre l'homme et le monde naturel sont lisibles grâce aux empreintes qu'il laisse sur le corps. Dans le cas du pacte diabolique, c'est la trace de l'Autre qui est laissée sur le corps, un sceau matériel qui prétend effacer et remplacer le signe symbolique et spirituel du baptême. Ce sceau a, à la fois, une fonction d'assujettissement et constitue le signe visible de l'intimité physique du contact.

Les historiens font remonter la genèse théologique de la notion de marque à un ensemble de traditions anciennes relatives aux "marques religieuses", fondées en particulier sur un solide dossier biblique. Du moment où la sorcellerie a été assimilée à une hérésie, le sabbat à une cérémonie effectivement célébrée, selon le principe d'un Satan "singe de Dieu", les sorciers sont apparus comme les prêtres d'une sorte d'anti-religion, les soldats d'une milice vouée à la destruction du peuple chrétien et marquée à l'instar des soldats romains ${ }^{25}$. À l'imitation donc des membres de l'armée du Christ, porteurs de la marque symbolique et invisible du baptême et, dans les cas du prêtre, du croisé, du saint et du mystique, d'emblèmes vestimentaires ou de stigmates certifiant leur appartenance à la divine cohorte, les combattants de la secte diabolique devaient porter l'empreinte diabolique, l'emblème de leur confrérie, l'antimarque attestant leur participation effective au projet transgressif et subversif de leur contre-culture ${ }^{26}$. Résultant d'un rituel d'initiation, le marquage devient le signe d'appartenance à la communauté, les démonologues s'accordant sur quelques données constantes - que la marque est un point insensible, généralement caché, qui ne saigne pas - en dépit de la très grande variabilité qui se manifeste dans tous les autres détails donnant corps à la croyance ${ }^{27}$.

sexe a suscités. Pour l'analyse de la construction démonologique, voir en particulier : Sophie HOUDARD, Les sciences du diable. Quatre discours sur la sorcellerie, Paris, Cerf, 1992.

25 Sur la notion de simia Dei : Jean CÉARD, "Le Diable singe de Dieu selon les démonologues des $\mathrm{XVI}^{\mathrm{e}}$ et $\mathrm{XVII}^{\mathrm{e}}$ siècles", in : Le Diable, Paris, Dervy, "Cahiers de l'Hermétisme", 1998, p. 31-45. Sur ce point : François DELPECH, "La 'marque' des sorcières : logique(s) de la stigmatisation diabolique", in Nicole Jacques-Chaquin et Maxime Préaud (dir.), Le sabbat des sorcières en Europe. XV $V^{e}$-XVIII siècles, Grenoble, Jérôme Million, 1993, p. 347368.

26 Alain Boureau en particulier a analysé l'émergence du principe de "sacrement satanique", où le diable détourne les deux principaux sacrements (baptême et eucharistie), c'està-dire construit un signe efficace, "un signe qui effectue ce qu'il figure" (Alain BOUREAU, Satan hérétique. Histoire de la démonologie (1280-1330), Paris, Odile Jacob, "Histoire", 2004, chapitre 2).

27 C'est pourquoi, il n'est guère de procès où les chirurgiens n'ont cherché ce point d'insensibilité, le trouvant du reste souvent - ce qui n'a rien d'étonnant si l'on se souvient que la grande hystérie crée chez les malades de telles zones insensibles, comme l'ont montré les travaux pionniers de Charcot et Freud. Cf. Jean-Martin ChARCoT et Paul Richer, Les 
Le solide dossier de citations bibliques, auquel ne cessent de renvoyer les textes démonologiques, ainsi que les traités théologiques et moraux, permet d'assimiler la marque à une tache. Les penseurs se fondent ici à la fois sur le "signe de Caïn",28 (Gn 4.15) et sur l'Apocalypse où les soldats de l'Antéchrist portent sur eux la "marque de la Bête" (Ap 13.16-17), antithèse de celle de l'Agneau (Ap 14.1) et de celle du "Cavalier fidèle et véridique" (Ap 19.1116). Globalement, les écrits canoniques multiplient les exemples qui imposent l'idée que l'homme est marqué dans sa chair, dans le corps qui ne laisse aucune cicatrice s'effacer. La rencontre entre l'homme et Dieu se traduit en particulier par un signe physique indélébile, comme dans deux passages cruciaux : le récit du combat de Jacob avec l'Ange (Gn 32.23-33) expose comment, au terme de la lutte jusqu'à l'aurore, Jacob reçoit un nouveau nom (Israël) et un stigmate physique : il boîte de la hanche, là où l'Ange l'a blessé $^{29}$. Dans le deuxième passage vétérotestamentaire (Ex 4.24-26) également, Dieu engage une lutte et n'hésite pas à blesser son adversaire. Jacob et Moïse, sur le chemin de leur expérience religieuse, sont marqués de leur rencontre; et le signe ne reste pas inscrit dans leur cœur ou dans la texture abstraite de l'intellect, mais se marque dans la chair.

Par analogie, les démonologues reconnaissent ainsi l'existence d'une manifestation corporelle d'appartenance à la secte démoniaque secrète. Le plus grand péché est bien de donner son corps, en même temps que son âme, au démon - d'où le fait que la marque devient élément primordial de la construction démonologique. Elle ne s'impose réellement qu'au cours des grandes chasses aux sorcières des $\mathrm{XVI}^{\mathrm{e}}$ et $\mathrm{XVII}^{\mathrm{e}}$ siècles ; elle s'impose donc, c'est un point essentiel, avant tout dans un contexte proprement judiciaire. Sa découverte ne suffisait pas à elle seule à décréter la peine de mort, mais autorisait seulement, si l'on peut dire, les magistrats à redoubler d'ardeur et à utiliser la torture en cas d'opiniâtreté de l'accusé. La recherche du stigmate maléfique s'effectuait sur un corps dénudé, entièrement rasé, sous le contrôle d'un chirurgien. Le principe de la marque transforme ainsi le mythe démonologique en certitude physique expérimentée par chacun - juge, "piqueur", public de l'exécution, voire l'accusé lui ou elle-même. Aux yeux des juges, la marque avait en effet au moins autant pour fonction d'affirmer la présence physique du diable que de prouver la culpabilité des prisonniers.

Le contexte judiciaire est donc primordial dans l'extension du phénomène et de son imaginaire, intimement lié au contrôle de l'État et à son appareil répressif; on mesure dès lors toute la richesse du concept et son potentiel de

démoniaques dans l'art, [1887], éd. Pierre Fédida, Georges Didi-Huberman, Paris, Macula, "Scènes", 1984.

28 Condamné à l'errance suite au meurtre d'Abel, Caïn ne peut être tué ; pour que nul ne l'ignore, "Yahvé mit un signe sur Cä̈n, afin que le premier venu ne le frappât point", signe d'exclusion autant que d'élection.

29 Pour l'interprétation de ce passage fameux : Jean-François MARQUET, "La lutte avec l'Ange", in L'Ange et l'Homme, Paris, Albin Michel, "Cahiers de l'Hermétisme", 1978, p. 221 238 ; Andréi PLESU, Actualité des Anges, trad. L. Hinckel, Paris, Buchet/Chastel, 2005, p. 219234. 
réinvestissement en particulier dans la nouvelle de Kafka. Les inquisiteurs et juges interrogent les conditions de visibilité du pacte diabolique, ils lisent et interprètent les signes laissés sur la peau du pactiseur, de la sorcière, du magicien. Carlo Ginzburg et Daniel Arasse rappellent tous deux à cet égard que le portrait et l'étude de la physiognomonie ont, depuis l'Antiquité, affaire avec l'identification judiciaire, la peau étant comprise comme une surface d'inscription (pigmentation, rides, tatouages, scarification) sur laquelle affleurent les signes identitaires, jusqu'à ces avatars de la société moderne que constituent l'anthropométrie ou le système de classification de Bertillon $^{30}$. C'est cette constellation originelle entre marque satanique, appareil judiciaire, volonté herméneutique et contrôle de l'État, qui se voit réinvestie par Kafka dans sa nouvelle, texte parmi d'autres irrémédiablement "hanté" 31 par ce fantasme, où survit une croyance ancienne qu'on aurait pu penser depuis longtemps révolue.

IV. L'ensemble du personnel de la nouvelle de Kafka, jusqu'au narrateur, est donc tributaire (prisonnier ?) de l'idée qu'on doit pouvoir lire ces corps et visages exposés, en particulier le corps supplicié sur la machine d'une extraordinaire complexité dessinée par l'ancien commandant, et commandée, comme par magie ou une force peut-être maléfique, par l'officier ${ }^{32}$. L'ensemble du dispositif est en particulier élaboré selon un principe de transparence absolue, de coïncidence parfaite entre la surface et la profondeur, permettant une opération judiciaire où la sentence et la "leçon" restent toujours parfaitement visibles et lisibles par le public : tout comme le doute est absent de la sentence - puisque la culpabilité du condamné est

30 Cf. Daniel ARASSE, Le portrait du Diable, p. 100-101 : "Dès lors que le Diable se donne à voir au travers d'indices démoniaques [...], le système de la représentation diabolique est promis, alors même que la croyance au Diable n'est plus dominante, à un ultime succès laïc et scientifique, au sein des enquêtes et des démonstrations triomphantes de l'anthropométrie judiciaire à la fin du $\mathrm{XIX}^{\mathrm{e}}$ siècle. Les séries de visages monstrueux, qui sont autant de visages de criminels, sont un avatar darwinien et scientiste de croyances anciennes et de la physiognomonie". Carlo Ginzburg, par ailleurs lui-même spécialiste de la chasse aux sorcières et des procès contre la secte satanique à la Renaissance (Carlo GINZBURG, Le Sabbat des sorcières, Paris, Gallimard, 1992), souligne également que le modèle épistémologique du "paradigme indiciaire" fonde aussi bien 1'histoire de l'art moderne que la méthode d'identification de Galton ou Bertillon.

31 C'est ce que souligne également l'historien Valentin Groebner en conclusion de sa passionnante étude : "In ihnen [moderne Dokumente] spuken weiterhin die alten Konzepte vom sichtbaren Zeichen auf der Haut, das seinen Träger unzweifelhaft identifizieren soll. Die komplexen modernen Systeme von Erfassung und Identifikation tragen ihre Vorläufer, die älteren bürokratischen Aufschreibetechniken und deren Kriterien der Unterscheidung, sozusagen in sich eingefroren" (Valentin Groebner, Der Schein der Person. Steckbrief, Ausweis und Kontrolle im Mittelalter, Munich, Beck, 2004, p. 161 ; je souligne).

32 Ce pouvoir surnaturel est en particulier visible dans la dernière partie du texte, lorsque l'officier s'offre en victime consentante à la machine, comme animée soudainement d'une volonté propre et s'actionnant seule : "so konnte es jetzt einen fast bestürzt machen, wie er mit ihr umging und wie sie gehorchte $[\ldots]$ Er hatte die Hand der Egge nur genähert, und sie hob und senkte sich $[\ldots]$ es war, als presse irgendeine große Macht" (p. 158-159). 
manifeste (unzweifelhaft) - le fonctionnement même de la machine vise à cette superposition et clarté absolues. Ainsi, dans la description initiale de l'appareil, l'officier insiste doublement sur le fait que la herse a été faite en verre pour que "chacun désormais [puisse] voir, à travers le verre, l'inscription s'exécuter dans le corps",33. Même prouesse, ingéniosité et minutie techniques en ce qui concerne l'inscription même, avec le double système d'aiguilles : "la longe inscrit, tandis que la courte projette de l'eau pour rincer le sang et maintenir l'inscription toujours lisible",34. Cet impératif de transparence est mimé dans le discours même de l'officier, avec ses appels récurrents et insistants sur la simplicité autant que clarté des procédures et fonctionnements ${ }^{35}$. La dialectique surface/profondeur est d'ailleurs variée au sein même de la nouvelle en rapport éloignement/proximité, là aussi annulé, comme lorsque l'officier tient à distance les dessins sacrés, mais tout en affirmant que le voyageur ne les verra pas moins "très bien" ${ }^{36}$.

Mais la parabole, tout en montrant le raffinement administratif et technologique qui peut être instauré pour réaliser ce fantasme de transparence absolue, en dénonce également les failles et limites irréductibles - à l'instar de la marque satanique des Inquisiteurs qui, bien qu'indubitable, n'en reste pas moins dissimulée, mouvante, insaisissable, transformant l'idéal de corps limpide en réalité d'un corps irrémédiablement opaque. Les brouillages, au sens propre, sont ainsi constants dans le système mis en place, mais aussi dans l'écriture de la nouvelle même. La herse en verre permet à chacun de vérifier l'exécution de la sentence, sauf que le public fait dorénavant défaut à la cérémonie qui auparavant avait lieu "devant des centaines d'yeux" Lorsque l'officier lui-même s'immole à la fin de la nouvelle, même si la machine fonctionne soudainement comme par magie comme il l'annonçait avec fierté au début ${ }^{38}$, elle n'en déraille pas moins complètement, se désossant inéluctablement : si les deux aiguilles avaient pour fonction de maintenir l'inscription toujours lisible, lors de ce "meurtre immédiat" (unmittelbarer Mord), non seulement la herse n'écrit pas, ne faisant que piquer, mais de surcroît "le sang coulait en cent ruisseaux, non mêlé d'eau"39. De même la méthode mécanique d'exécution, observée de près par l'officier de manière à recueillir "l'expression transfigurée du visage martyrisé", cette

33 P. 96 ; "nun kann jeder durch das Glas sehen, wie sich die Inschrift im Körper vollzieht" (p. 139).

34 P. 96 ; "Die lange schreibt nämlich, und die kurze spritzt Wasser aus, um das Blut abzuwaschen und die Schrift immer klar zu erhalten" (p. 139).

35 P. 94-95; "das war alles sehr einfach", "Ist Ihnen das klar?” (p. 138).

36 Cf. p. 141 : "ich zeige sie Ihnen aus dieser Entfernung, dann werden Sie alles gut sehen können". Voir aussi p. 156 : "Der Reisende beugte sich so tief über das Papier, dass der Offizier aus Angst von einer Berührung es weiter entfernte" (le texte ici combine les dialectiques profondeur/surface (tief) et proximité/distance).

37 P. 106 ; "vor hunderten Augen" (p. 147).

38 Par exemple p. 132 : "von jetzt aber arbeitet der Apparat ganz allein".

39 P. 123 ; "Das Blut floss in hundert Strömen, nicht mit Wasser vermischt" (p. 160). 
illumination qui, de manière significative "débute autour des yeux" complètement lors de la dernière exécution, le sacrifice de l'officier. Le voyageur, lorsqu'il veut parachever l'œuvre de mort en faisant basculer le cadavre dans la fosse, en voit alors le visage, y lisant l'absence de "transfiguration" annoncée : "il était tel que du vivant de l'officier; on ne découvrait pas signe de la grâce promise" $" 41$.

Cet échec programmé est d'ailleurs indiqué d'entrée de jeu. Nous avons déjà parlé du brouillage opéré entre profondeur et surface (ou distance et proximité) ; les deux niveaux paraissent éminemment indistincts, la profondeur ne recélant pas nécessairement plus de vérité et de valeur que la surface ; il paraît dès lors vain de vouloir la faire affleurer, par la force et même la torture, par le biais de l'herméneutique autant que de la machine. D'autre part, ce brouillage est également inhérent au principe même de l'écriture, qui répond à des impératifs contradictoires à la fois de lisibilité et de secret, posant mais sans les distinguer les limites du visible et du cachée ${ }^{22}$. C'est en particulier ce qui ressort des deux passages où le voyageur est enjoint de "lire" l'écriture de l'ancien commandant, mais en vain. Cette écriture labyrinthique, hiéroglyphique et pourtant indubitable, surchargée d'enjolivures, cette inscription qu'il n'est pas facile de déchiffrer "avec ses yeux", mais que le supplicié peut pourtant déchiffrer "avec ses plaies", apprenant la vérité "à son corps défendant" comme écran. Les deux échanges entre l'officier et le voyageur mettent ainsi en lumière le caractère irréconciliable de ces injonctions paradoxales, entre une écriture parfaitement "nette" et claire et pourtant totalement énigmatique, impossible à déchiffrer, posant d'ailleurs la question de savoir comment on peut "lire" ce qui est proprement illisible ${ }^{44}$. Toute l'ironie du texte tient non

40 P. 106 et 100 ; “Ausdruck der Verklärung von dem gemarterten Gesicht", "Um die Augen beginnt es. Von hier aus verbreitet es sich" (p. 147 et 142).

41 P. 124 ; "Es war, wie es im Leben gewesen war ; kein Zeichen der versprochenen Erlösung war zu entdecken" (p. 161). Je souligne.

42 L'ensemble de cette problématique est à mettre en rapport avec le dernier ouvrage du sociologue Luc Boltanski (Enigmes et complots - Une enquête à propos d'enquêtes, Paris, Gallimard, "Essais", 2012). Il y analyse l'émergence concomitante du roman policier, du roman d'espionnage, de la psychiatrie et de la sociologie. La raison en serait la conjoncture nouvelle que créent de profonds changements dans la façon dont est instaurée la réalité sociale, en particulier en lien avec la mise en place de l'État-nation auquel on doit le projet d'organiser et d'unifier la réalité pour une population et sur un territoire, projet proprement démiurgique. La figure du complot focaliserait des soupçons qui concernent l'exercice du pouvoir, Boltanski montrant comment s'échafaudent des ontologies politiques qui tablent sur une réalité doublement distribuée : à une réalité officielle, mais de surface et illusoire, s'oppose une réalité profonde, cachée, menaçante, officieuse, qui serait bien plus réelle. On retrouve donc la dialectique surface/profondeur et son lien consubstantiel avec l'appareil d'État, notamment judiciaire, indissociable d'une façon de problématiser la réalité et de travailler les contradictions qui l'habitent.

43 P. 100 et 92: "Es ist nicht leicht, die Schrift mit den Augen zu entziffern; unser Mann entziffert sie aber mit seinen Wunden", "Er erfährt es ja auf seinem Leib" (p. 142 et 136).

44 Cf. p. 141 et p. 155-156: "Lesen Sie', sagte der Offizier. 'Ich kann nicht', sagte der Reisende. 'Es ist doch deutlich', sagte der Offizier. 'Es ist sehr kunstvoll', sagte der Reisende 
seulement en ces échanges absurdes, mais aussi dans le mimétisme d'un narrateur qui lui aussi se base sur cet idéal de transparence, comme pour mieux, à son insu, le démentir. L'unique "évidence" qu'il finit par établir est précisément que le voyageur "n'avait toujours rien pu lire", tout comme la seule chose "manifeste", sous forme de révélation paradoxale (Offenbarung), est seulement le dysfonctionnement, et même le fait que la machine se désagrège, tout le reste n'étant qu'"illusion" (Täuschung) ${ }^{45}$.

À l'opposé de l'ambition de clarté (et de justice infaillible, fondée en droit et immuablement valable), l'ensemble du texte ne fait qu'insister sur les blocages et opacités au sein même des univers de signes construits et offerts par les personnages. L'officier a conscience qu'il est "impossible de faire comprendre aujourd'hui ce qu'était ce temps-là" tout comme le voyageur ne peut lui faire "comprendre" sa propre position ${ }^{46}$ - les exemples extrêmes d'impossible compréhension étant incarnés par le soldat et le condamné. Cette opacité irréductible, face à un mécanisme qui revendique pourtant la transparence parfaite, va jusqu'à contaminer le lecteur lui-même, placé dans une position inconfortable face à un récit d'une violence insoutenable, mais irréductiblement mystérieux. Kafka pousse ainsi à sa limite la logique d'une organisation sociale si parfaitement "cohérente" (in sich geschlossen) qu'elle en devient irrationnelle, d'un appareil d'État qui ne s'est jamais départi des croyances "magiques", pourtant irréalisables ${ }^{47}$.

La machine avec son processus d'écriture corporelle, tout comme la nouvelle dans son ensemble, se retrouve donc à l'image du stigmate satanique dans son caractère irréductiblement paradoxal : à la fois indubitables et invisibles, la peau (et le texte) donnent à voir, tout en masquant, le corps (humain, social, poétique) étant bien pensé comme une construction essentiellement sémiotique. L'anatomie politique, métaphysique, religieuse

ausweichend, 'aber ich kann es nicht entziffern”" ; “Lesen Sie', sagte er. 'Ich kann diese Blätter nicht lesen'. 'Sehen Sie das Blatt doch genau an', sagte der Offizier. [...] Nun begann der Offizier die Aufschrift zu buchstabieren und dann las er sie noch einmal im Zusammenhang. 'Sei gerecht ! - heißt es', sagte er, 'jetzt können Sie es doch lesen"'.

45 P. 117: "aber es war klar, dass er es noch immer nicht hatte lesen können" (p. 156). Cf. p. 160 : "die Maschine ging offenbar in Trümmer ; ihr ruhiger Gang war eine Täuschung" (p. 160); je souligne.

46 P. 107 et 110 ; "es ist unmöglich, jene Zeiten heute begreiflich zu machen", "Begriff es schon der Offizier? Nein, er begriff noch nicht" (p. 148 et 150). On a ici une troisième variation de la dialectique surface/profondeur, sous forme de la tension distance temporelle/présent, qui se heurte à un insurmontable effet-retard, décalage, interdisant une nouvelle fois la réalisation de la coïncidence parfaite. De surcroît, cela se traduit également par l'image de l'"emprise", de la "main mise" (begreifen), central à notre problématique tout comme à la nouvelle.

47 Cela permet aussi de lever ce paradoxe auquel se heurtent nombre de critiques de la nouvelle de Kafka, qui trouvent inconciliables la dimension la plus moderne du texte, en particulier technologique, et son inscription dans la sphère la plus archaïque. Or la contradiction n'est encore une fois qu'apparente, Kafka ne fait que pousser à sa limite la logique "archaïque" présente dans la modernité la plus radicale - tout comme Ginzburg a pu montrer que l'opposition rationalisme / irrationalisme est stérile et qu'un même paradigme est au fondement aussi bien de deux pseudo-sciences, comme la divination et la physiognomonie, que de deux sciences attestées comme le droit et la médecine. 
de ce corps mis en scène montre ainsi que le "démoniaque" couvre par excellence toutes les surfaces, les visibles et les cachées, bref, que le diable est bien dans les détails. D'où aussi ces constants phénomènes de confusion, de brouillage ${ }^{48}$ et les ambiguïtés herméneutiques qui en résultent : il est là où on le voit, mais rien ne dit qu'il est absent dès qu'on ne l'aperçoit plus, de sorte qu'il est maître des multiples accès qui permettent de voir, de vérifier et de dire où est la vérité. Il commande, en fait, tous les chemins de l'équivoque, $\mathrm{du}$ doute et de l'erreur. Ces jeux d'apparitions et de disparitions, d'éblouissements et de déceptions de l'œil se heurtent pourtant toujours à une absence perpétuelle, à une "empreinte" insensible, inassignable souvent, constamment insaisissable, qui interdit par là même un savoir stable. Ce caractère vagabond interdit de "localiser" définitivement ce démoniaque, en surface autant qu'en profondeur, au plus près comme au plus éloigné, dedans et dehors ${ }^{49}$.

Suite à la chasse aux sorcières, qui reposait sur son corps de savoir propre, parfaitement cohérent et non moins cruel que l'appareil mis en scène dans la Colonie pénitentiaire, a émergé un nouveau corpus de textes pour remplacer le précédent. Mais en chassant le démoniaque par la porte, cela n'a permis que de le faire rentrer par la fenêtre, les savants élaborant des discours visant à supplanter la raison en démonologie qui définissait les limites du naturel et du surnaturel, de ce qui est et de ce qui paraît être, du visible et du caché. Distribuant différemment ces catégories, la modernité a imposé d'autres langages et d'autres savoirs, d'autres croyances et d'autres cultures, mais qui n'en placent pas moins en leur cœur la question des propriétés occultes, des principaux prestiges diaboliques, même si dorénavant ils ne portent plus ce nom. Enfin, si le corps et la peau humaine sont par excellence un texte ambigu, qui appelle l'interprétation, un corps marqué sur lequel on pourrait lire à livre ouvert la biographie de l'être, un corps qui montre l'imprégnation à l'aide de signes et de traces ostensibles mais qui demandent pourtant à être révélés, la question nodale reste bien celle de la place que doit dès lors assumer l'observateur - le chercheur en voyages d'études, prisonnier de ses conceptions européennes, mais tout autant chacun de nous lecteurs, toujours plus ou moins complices des exactions, pratiques et récits proposés.

\footnotetext{
48 On pensera également dans ce contexte aux confusions dans les rôles occupés par les personnages - l'officier est à la fois juge, président du tribunal, chef-mécanicien, bourreau et victime sacrificielle, à l'instar de l'ancien commandant, tout à la fois "soldat, juge, technicien, chimiste, dessinateur". À aucun personnage n'est assignée une position unique ni stable.

49 Telles sont bien les tensions du démoniaque qui sont constamment repensées par les savants. Ainsi l'alternative dedans/dehors change radicalement de statut avec la promotion du "naturel", c'est-à-dire d'une autre tradition (scientifique, médicale) à partir du XVII' siècle. Cf. Michel Foucault, "Les Déviations religieuses et le savoir médical", in Jacques LE GofF (dir.), Hérésies et Sociétés dans l'Europe pré-industrielle, XI ${ }^{e}-X V I I I^{e}$ siècle, Paris, Mouton, 1968, p. 19-25; Hervé Guillemain, "Déments ou démons ? L'exorcisme face aux sciences psychiques (XIX $-\mathrm{XX}^{\mathrm{e}}$ siècles)", in Revue d'histoire de l'Église de France, Paris, t. 87, n 219 , juil.-déc. 2001, p. 439-471.
} 